\title{
ON THE IMPORTANCE OF SECOND-ORDER RESPONSE SENSITIVITIES TO NUCLEAR DATA IN REACTOR PHYSICS UNCERTAINTY ANALYSIS
}

\author{
Dan G. Cacuci ${ }^{1}$ \\ ${ }^{1}$ University of South Carolina \\ 300 Main Street, Columbia, SC 29208, USA \\ cacuci@cec.sc.edu
}

\begin{abstract}
This invited keynote presentation compares the relative importance of $1^{\text {st }}$-order versus $2^{\text {nd }}$ order sensitivities of the leakage response of an OECD/NEA benchmark (polyethylenereflected plutonium sphere) to the nuclear data characterizing this benchmark. The imprecisely known parameters underlying the neutron transport computational model for this benchmark include 180 group-averaged total microscopic cross sections, 21600 groupaveraged scattering microscopic cross sections, 60 parameters describing the fission process, 30 parameters describing the fission spectrum, 10 parameters describing the system's sources, and 6 isotopic number densities. Thus, this benchmark comprises 21886 1st-order sensitivities of the leakage response with respect to the model parameters, and 478,996,996 2nd-order sensitivities, of which 239,509,441 are distinct. The exact deterministic computation of all of these 1st- and 2nd-order sensitivities was made possible by the application of the Second-Order Adjoint Sensitivity Analysis Methodology (2 $\left.2^{\text {nd }}-A S A M\right)$ developed by Cacuci. Thousands (out of the 32400 elements) of the $2^{\text {nd }}$-order sensitivities of the leakage response with respect to the total cross sections turned out to be significantly larger than the largest corresponding 1st-order sensitivities, contrary to some previously held beliefs in the reactor physics community. Hence, it will be shown that neglecting the 2ndorder sensitivities to total cross sections would cause very large non-conservative errors by under-reporting the response's variance and expected value. The 2nd-order sensitivities also cause the response distribution to be skewed towards positive values relative to the expected value, which, in turn, is significantly larger than the computed value of the leakage response. The result presented in this paper also underscore the need for obtaining reliable cross section covariance data, which are not available at this time.
\end{abstract}

KEYWORDS: polyethylene-reflected plutonium sphere (PERP) benchmark; $1^{\text {st }}$-and $2^{\text {nd }}$-order sensitivities; total cross sections; expected value, variance and skewness of PERP response;

\section{INTRODUCTION}

Several important subcritical fundamental physics benchmarks within the Nuclear Energy Agency (NEA) International Criticality Safety Benchmark Evaluation Project (ICSBEP) Handbook [1] use as particle source a $4.5 \mathrm{~kg}$ alpha-phase plutonium sphere colloquially known as the "BeRP ball," which was originally constructed at Los Alamos National Laboratory in 1980 [2] for conducting experiments aimed at estimating the reactivity worth of beryllium reflectors (hence the acronym "BeRP" for "berylliumreflected plutonium"). Subsequent experiments have used the BeRP ball as the source for other subcritical 
experiments (accepted in the ICSBEP book as fundamental physics benchmarks) using tungsten, nickel or polyethylene reflectors around the BeRP ball. In their computational evaluation of neutron multiplicity measurements for the "polyethylene-reflected BeRP" ball, Miller et al. [3] showed that the computational results disagreed significantly with the corresponding measurements of neutron multiplicity and concluded that "only a subtle variation in the value of the average number of neutrons produced per fission" for 239Pu was able to improve the simulations of the plutonium sphere..."

However, the "polyethylene-reflected BeRP ball" benchmark contains many imprecisely known nuclear data parameters, in addition to those that were incidentally investigated in [3]. Therefore, the sensitivities to all of the benchmark's nuclear data would need to be quantified in order to assess the uncertainties in the computed responses that are, in turn, compared to measurements. For this purpose, Cacuci's $2^{\text {nd }}-$ ASAM for subcritical systems [4] has been applied to compute all of the $1^{\text {st }}$ - and $2^{\text {nd }}$-order sensitivities of the leakage response of this benchmark with respect to the nuclear data that characterizes this benchmark. This work summarizes the most significant results obtained for the $1^{\text {st }}$ - and $2^{\text {nd }}$-order sensitivities of the "polyethylene-reflected BeRP ball" benchmark's leakage response with respect to the benchmark's groupaveraged isotopic total cross sections, highlighting the finding that many of the $2^{\text {nd }}$-order sensitivities are much larger than the corresponding $1^{\text {st }}$-order sensitivities. The $2^{\text {nd }}$-order sensitivities shift the leakage response's expected value significantly away from the computed value of the leakage response, and cause asymmetries in the response distribution. Furthermore, the effects of the $2^{\text {nd }}$-order leakage response sensitivities with respect to the isotopic total cross sections on the leakage responses expected value, variance, and skewness, are much larger than the corresponding effects stemming from the 1st-order sensitivities.

\section{LARGEST FIRST- AND SECOND-ORDER SENSITIVITIES OF THE PERP BENCHMARK'S LEAKAGE WITH RESPECT TO PERP'S NUCLEAR DATA}

The "polyethylene-reflected plutonium sphere," which will henceforth be abbreviated as "PERP," contains two materials designated as "material 1" and "material 2". Material 1 (core) contains the following isotopes: ${ }^{239} \mathrm{Pu},{ }^{240} \mathrm{Pu},{ }^{69} \mathrm{Ga},{ }^{71} \mathrm{Ga}$. Material 2 (reflector) contains the following isotopes: $\mathrm{C}$ and ${ }^{1} \mathrm{H}$. The neutron flux distribution within the PERP benchmark is computed by using the PARTISN [5] multigroup discrete ordinates particle transport code. The specific computations in this work were performed using a $\mathrm{P}_{3}$ Legendre expansion of the scattering cross section, an angular quadrature of $\mathrm{S}_{256}$, and a fine-mesh spacing of $0.005 \mathrm{~cm}$ (comprising 759 meshes for the plutonium sphere of radius of 3.794 $\mathrm{cm}$, and 762 meshes for the polyethylene shell of thickness of $3.81 \mathrm{~cm}$ ). The PARTISN [5] computations used the MENDF71X [6] 618-group cross section data collapsed to $G=30$ energy groups. The details of these computations are presented in [7]. Since the total leakage is physically more meaningful than count rates (because it does not depend on the detector configuration), the total leakage of the PERP benchmark, which will be denoted as $L(\boldsymbol{\alpha})$, will be considered as the response of interest for sensitivity analysis.

The leakage response displayed the largest $1^{\text {st }}$ - and $2^{\text {nd }}$-order sensitivities with respect to the group-averaged total microscopic cross sections for ${ }^{1} \mathrm{H}$, as presented in Tables 1 and 2 (see Ref. 7 for complete results for all isotopes).

Table 1. First-order relative sensitivities, $\left(\partial L / \partial \sigma_{t, i=6}^{g}\right)\left(\sigma_{t, i=6}^{g} / L\right), g=1, \ldots, 30$, of the leakage to the total cross sections of ${ }^{1} \mathrm{H}$ (isotope $i=6$ )

\begin{tabular}{cc|cccccccc}
\hline $\mathbf{g}$ & $\mathbf{1}^{\text {st }}-$ order & $\mathbf{G}$ & $\mathbf{1}^{\text {st }}-$ order & $\mathbf{g}$ & $\mathbf{1}^{\text {st }}$-order & $\mathbf{g}$ & $\mathbf{1}^{\text {st }}$-order & $\mathbf{G}$ & $\mathbf{1}^{\text {st }}-$ order \\
\hline $1-6$ & $<-0.01$ & 11 & -0.19 & 16 & -1.16 & 21 & -9.69 & 26 & -0.65 \\
7 & -0.07 & 12 & -0.44 & 17 & -1.17 & 22 & -0.92 & 27 & -0.58 \\
8 & -0.09 & 13 & -0.52 & 18 & -1.14 & 23 & -0.89 & 28 & -0.55 \\
9 & -0.14 & 14 & -0.57 & 19 & -1.09 & 24 & -0.75 & 29 & -0.55 \\
10 & -0.17 & 15 & -0.58 & 20 & -1.03 & 25 & -0.71 & 30 & $\mathbf{- 9 . 3 6 6}$ \\
\hline
\end{tabular}


Table 2. Selected $2^{\text {nd }}$-order relative sensitivities $\left(\partial^{2} L / \partial \sigma_{t, i=6}^{g} \partial \sigma_{t, k=6}^{g}\right)\left(\sigma_{t, i=6}^{g} \sigma_{t, k=6}^{g} / L\right), g=1, \ldots, 30$, having values greater than 1.0 , for ${ }^{1} \mathrm{H}$.

\begin{tabular}{cccccccccccc}
\hline & $\mathrm{g}^{\prime}=\mathbf{1 2}$ & $\mathbf{1 3}$ & $\mathbf{1 4}$ & $\mathbf{1 5}$ & $\mathbf{1 6}$ & $\mathbf{1 7}$ & $\mathbf{1 8}$ & $\mathbf{1 9}$ & $\mathbf{2 0}$ & $\mathbf{2 1}$ & $\mathbf{2 2}$ \\
$\mathrm{g}=12$ & 0.653 & 0.315 & 0.340 & 0.356 & 0.740 & 0.763 & 0.751 & 0.725 & 0.688 & 0.648 & 0.597 \\
13 & 0.315 & 0.974 & 0.471 & 0.471 & 0.976 & $\mathbf{1 . 0 0 5}$ & 0.988 & 0.953 & 0.904 & 0.851 & 0.784 \\
14 & 0.340 & 0.471 & $\mathbf{1 . 2 6 1}$ & 0.579 & $\mathbf{1 . 1 5 8}$ & $\mathbf{1 . 1 9 2}$ & $\mathbf{1 . 1 7 2}$ & $\mathbf{1 . 1 3 0}$ & $\mathbf{1 . 0 7 2}$ & $\mathbf{1 . 0 0 9}$ & 0.930 \\
15 & 0.356 & 0.471 & 0.579 & $\mathbf{1 . 3 9 1}$ & $\mathbf{1 . 2 5 5}$ & $\mathbf{1 . 2 7 7}$ & $\mathbf{1 . 2 5 5}$ & $\mathbf{1 . 2 1 0}$ & $\mathbf{1 . 1 4 8}$ & $\mathbf{1 . 0 8 1}$ & 0.996 \\
16 & 0.740 & 0.976 & $\mathbf{1 . 1 5 8}$ & $\mathbf{1 . 2 5 5}$ & $\mathbf{4 . 4 6 1}$ & $\mathbf{2 . 7 0 0}$ & $\mathbf{2 . 6 4 7}$ & $\mathbf{2 . 5 5 3}$ & $\mathbf{2 . 4 2 1}$ & $\mathbf{2 . 2 8 0}$ & $\mathbf{2 . 1 0 0}$ \\
17 & 0.763 & $\mathbf{1 . 0 0 5}$ & $\mathbf{1 . 1 9 2}$ & $\mathbf{1 . 2 7 7}$ & $\mathbf{2 . 7 0 0}$ & $\mathbf{4 . 8 5 3}$ & $\mathbf{2 . 7 8 9}$ & $\mathbf{2 . 6 8 4}$ & $\mathbf{2 . 5 4 6}$ & $\mathbf{2 . 3 9 8}$ & $\mathbf{2 . 2 0 9}$ \\
18 & 0.751 & 0.988 & $\mathbf{1 . 1 7 2}$ & $\mathbf{1 . 2 5 5}$ & $\mathbf{2 . 6 4 7}$ & $\mathbf{2 . 7 8 9}$ & $\mathbf{4 . 8 2 8}$ & $\mathbf{2 . 6 8 9}$ & $\mathbf{2 . 5 4 6}$ & $\mathbf{2 . 3 9 9}$ & $\mathbf{2 . 2 1 0}$ \\
19 & 0.725 & 0.953 & $\mathbf{1 . 1 3 0}$ & $\mathbf{1 . 2 1 0}$ & $\mathbf{2 . 5 5 3}$ & $\mathbf{2 . 6 8 4}$ & $\mathbf{2 . 6 8 9}$ & $\mathbf{4 . 6 1 9}$ & $\mathbf{2 . 4 9 8}$ & $\mathbf{2 . 3 4 9}$ & $\mathbf{2 . 1 6 5}$ \\
20 & 0.688 & 0.904 & $\mathbf{1 . 0 7 2}$ & $\mathbf{1 . 1 4 8}$ & $\mathbf{2 . 4 2 1}$ & $\mathbf{2 . 5 4 6}$ & $\mathbf{2 . 5 4 6}$ & $\mathbf{2 . 4 9 8}$ & $\mathbf{4 . 2 8 4}$ & $\mathbf{2 . 2 6 6}$ & $\mathbf{2 . 0 8 5}$ \\
21 & 0.648 & 0.851 & $\mathbf{1 . 0 0 9}$ & $\mathbf{1 . 0 8 1}$ & $\mathbf{2 . 2 8 0}$ & $\mathbf{2 . 3 9 8}$ & $\mathbf{2 . 3 9 9}$ & $\mathbf{2 . 3 4 9}$ & $\mathbf{2 . 2 6 6}$ & $\mathbf{3 . 9 3 7}$ & $\mathbf{2 . 0 0 4}$ \\
22 & 0.597 & 0.784 & 0.930 & 0.996 & $\mathbf{2 . 1 0 0}$ & $\mathbf{2 . 2 0 9}$ & $\mathbf{2 . 2 1 0}$ & $\mathbf{2 . 1 6 5}$ & $\mathbf{2 . 0 8 5}$ & $\mathbf{2 . 0 0 4}$ & $\mathbf{3 . 5 1 5}$ \\
23 & 0.554 & 0.728 & 0.863 & 0.924 & $\mathbf{1 . 9 4 9}$ & $\mathbf{2 . 0 5 0}$ & $\mathbf{2 . 0 5 1}$ & $\mathbf{2 . 0 1 0}$ & $\mathbf{1 . 9 3 6}$ & $\mathbf{1 . 8 5 7}$ & $\mathbf{1 . 7 6 0}$ \\
24 & 0.502 & 0.659 & 0.782 & 0.837 & $\mathbf{1 . 7 6 7}$ & $\mathbf{1 . 8 5 8}$ & $\mathbf{1 . 8 5 9}$ & $\mathbf{1 . 8 2 2}$ & $\mathbf{1 . 7 5 5}$ & $\mathbf{1 . 6 8 4}$ & $\mathbf{1 . 5 9 3}$ \\
25 & 0.477 & 0.627 & 0.742 & 0.795 & $\mathbf{1 . 6 7 7}$ & $\mathbf{1 . 7 6 4}$ & $\mathbf{1 . 7 6 4}$ & $\mathbf{1 . 7 2 9}$ & $\mathbf{1 . 6 6 6}$ & $\mathbf{1 . 5 9 9}$ & $\mathbf{1 . 5 1 2}$ \\
26 & 0.440 & 0.577 & 0.684 & 0.733 & $\mathbf{1 . 5 4 6}$ & $\mathbf{1 . 6 2 5}$ & $\mathbf{1 . 6 2 6}$ & $\mathbf{1 . 5 9 4}$ & $\mathbf{1 . 5 3 5}$ & $\mathbf{1 . 4 7 4}$ & $\mathbf{1 . 3 9 4}$ \\
27 & 0.393 & 0.516 & 0.611 & 0.655 & $\mathbf{1 . 3 8 1}$ & $\mathbf{1 . 4 5 2}$ & $\mathbf{1 . 4 5 3}$ & $\mathbf{1 . 4 2 4}$ & $\mathbf{1 . 3 7 2}$ & $\mathbf{1 . 3 1 7}$ & $\mathbf{1 . 2 4 6}$ \\
28 & 0.369 & 0.484 & 0.574 & 0.615 & $\mathbf{1 . 2 9 6}$ & $\mathbf{1 . 3 6 3}$ & $\mathbf{1 . 3 6 3}$ & $\mathbf{1 . 3 3 6}$ & $\mathbf{1 . 2 8 7}$ & $\mathbf{1 . 2 3 6}$ & $\mathbf{1 . 1 6 9}$ \\
29 & 0.372 & 0.487 & 0.576 & 0.617 & $\mathbf{1 . 3 0 0}$ & $\mathbf{1 . 3 6 7}$ & $\mathbf{1 . 3 6 7}$ & $\mathbf{1 . 3 4 0}$ & $\mathbf{1 . 2 9 0}$ & $\mathbf{1 . 2 3 8}$ & $\mathbf{1 . 1 7 1}$ \\
30 & $\mathbf{6 . 4 3 2}$ & $\mathbf{8 . 4 2 4}$ & $\mathbf{9 . 9 7}$ & $\mathbf{1 0 . 6 7}$ & $\mathbf{2 2 . 4 8}$ & $\mathbf{2 3 . 6 2}$ & $\mathbf{2 3 . 6 2}$ & $\mathbf{2 3 . 1 5}$ & $\mathbf{2 2 . 2 9}$ & $\mathbf{2 1 . 4 0}$ & $\mathbf{2 0 . 2 4}$ \\
\hline
\end{tabular}

Table 3. Continuation of Table 2.

\begin{tabular}{ccccccccc}
\hline & $\mathbf{g}^{\prime}=\mathbf{2 3}$ & $\mathbf{2 4}$ & $\mathbf{2 5}$ & $\mathbf{2 6}$ & $\mathbf{2 7}$ & $\mathbf{2 8}$ & $\mathbf{2 9}$ & $\mathbf{3 0}$ \\
\hline $\mathrm{g}=12$ & 0.554 & 0.502 & 0.477 & 0.440 & 0.393 & 0.369 & 0.372 & $\mathbf{6 . 4 3 2}$ \\
13 & 0.728 & 0.659 & 0.627 & 0.577 & 0.516 & 0.484 & 0.487 & $\mathbf{8 . 4 2 4}$ \\
14 & 0.863 & 0.782 & 0.742 & 0.684 & 0.611 & 0.574 & 0.576 & $\mathbf{9 . 9 6 8}$ \\
15 & 0.924 & 0.837 & 0.795 & 0.733 & 0.655 & 0.615 & 0.617 & $\mathbf{1 0 . 6 7}$ \\
16 & $\mathbf{1 . 9 4 9}$ & $\mathbf{1 . 7 6 7}$ & $\mathbf{1 . 6 7 7}$ & $\mathbf{1 . 5 4 6}$ & $\mathbf{1 . 3 8 1}$ & $\mathbf{1 . 2 9 6}$ & $\mathbf{1 . 3 0 0}$ & $\mathbf{2 2 . 4 8}$ \\
17 & $\mathbf{2 . 0 5 0}$ & $\mathbf{1 . 8 5 8}$ & $\mathbf{1 . 7 6 4}$ & $\mathbf{1 . 6 2 5}$ & $\mathbf{1 . 4 5 2}$ & $\mathbf{1 . 3 6 3}$ & $\mathbf{1 . 3 6 7}$ & $\mathbf{2 3 . 6 2}$ \\
18 & $\mathbf{2 . 0 5 1}$ & $\mathbf{1 . 8 5 9}$ & $\mathbf{1 . 7 6 4}$ & $\mathbf{1 . 6 2 6}$ & $\mathbf{1 . 4 5 3}$ & $\mathbf{1 . 3 6 3}$ & $\mathbf{1 . 3 6 7}$ & $\mathbf{2 3 . 6 2}$ \\
19 & $\mathbf{2 . 0 1 0}$ & $\mathbf{1 . 8 2 2}$ & $\mathbf{1 . 7 2 9}$ & $\mathbf{1 . 5 9 4}$ & $\mathbf{1 . 4 2 4}$ & $\mathbf{1 . 3 3 6}$ & $\mathbf{1 . 3 4 0}$ & $\mathbf{2 3 . 1 5}$ \\
20 & $\mathbf{1 . 9 3 6}$ & $\mathbf{1 . 7 5 5}$ & $\mathbf{1 . 6 6 6}$ & $\mathbf{1 . 5 3 5}$ & $\mathbf{1 . 3 7 2}$ & $\mathbf{1 . 2 8 7}$ & $\mathbf{1 . 2 9 0}$ & $\mathbf{2 2 . 2 9}$ \\
21 & $\mathbf{1 . 8 5 7}$ & $\mathbf{1 . 6 8 4}$ & $\mathbf{1 . 5 9 9}$ & $\mathbf{1 . 4 7 4}$ & $\mathbf{1 . 3 1 7}$ & $\mathbf{1 . 2 3 6}$ & $\mathbf{1 . 2 3 8}$ & $\mathbf{2 1 . 4 0}$ \\
22 & $\mathbf{1 . 7 6 0}$ & $\mathbf{1 . 5 9 3}$ & $\mathbf{1 . 5 1 2}$ & $\mathbf{1 . 3 9 4}$ & $\mathbf{1 . 2 4 6}$ & $\mathbf{1 . 1 6 9}$ & $\mathbf{1 . 1 7 1}$ & $\mathbf{2 0 . 2 4}$ \\
23 & $\mathbf{3 . 1 7 7}$ & $\mathbf{1 . 5 2 1}$ & $\mathbf{1 . 4 4 0}$ & $\mathbf{1 . 3 2 8}$ & $\mathbf{1 . 1 8 7}$ & $\mathbf{1 . 1 1 4}$ & $\mathbf{1 . 1 1 6}$ & $\mathbf{1 9 . 2 8}$ \\
24 & $\mathbf{1 . 5 2 1}$ & $\mathbf{2 . 7 9 2}$ & $\mathbf{1 . 3 5 8}$ & $\mathbf{1 . 2 4 9}$ & $\mathbf{1 . 1 1 7}$ & $\mathbf{1 . 0 4 8}$ & $\mathbf{1 . 0 4 9}$ & $\mathbf{1 8 . 1 3}$ \\
25 & $\mathbf{1 . 4 4 0}$ & $\mathbf{1 . 3 5 8}$ & $\mathbf{2 . 6 0 4}$ & $\mathbf{1 . 2 1 4}$ & $\mathbf{1 . 0 8 2}$ & $\mathbf{1 . 0 1 6}$ & $\mathbf{1 . 0 1 7}$ & $\mathbf{1 7 . 5 8}$ \\
26 & $\mathbf{1 . 3 2 8}$ & $\mathbf{1 . 2 4 9}$ & $\mathbf{1 . 2 1 4}$ & $\mathbf{2 . 3 4 9}$ & $\mathbf{1 . 0 3 7}$ & 0.971 & 0.972 & $\mathbf{1 6 . 7 9}$ \\
27 & $\mathbf{1 . 1 8 7}$ & $\mathbf{1 . 1 1 7}$ & $\mathbf{1 . 0 8 2}$ & $\mathbf{1 . 0 3 7}$ & $\mathbf{2 . 0 3 9}$ & 0.913 & 0.912 & $\mathbf{1 5 . 7 6}$ \\
28 & $\mathbf{1 . 1 1 4}$ & $\mathbf{1 . 0 4 8}$ & $\mathbf{1 . 0 1 6}$ & 0.971 & 0.913 & $\mathbf{1 . 8 8 5}$ & 0.888 & $\mathbf{1 5 . 3 0}$ \\
29 & $\mathbf{1 . 1 1 6}$ & $\mathbf{1 . 0 4 9}$ & $\mathbf{1 . 0 1 7}$ & 0.972 & 0.912 & 0.888 & $\mathbf{1 . 8 9 1}$ & $\mathbf{1 5 . 3 9}$ \\
30 & $\mathbf{1 9 . 2 8}$ & $\mathbf{1 8 . 1 3}$ & $\mathbf{1 7 . 5 8}$ & $\mathbf{1 6 . 7 9}$ & $\mathbf{1 5 . 7 6}$ & $\mathbf{1 5 . 3 0}$ & $\mathbf{1 5 . 3 9}$ & $\mathbf{4 2 9 . 6}$ \\
\hline
\end{tabular}




\section{UNCERTAINTY QUANTIFICATION}

Correlations among the group total cross sections are not available for the PERP benchmark. Two extreme situations can be considered, as follows: (i) all cross sections are uncorrelated, which will be considered in Section 3.1 below; (ii) all cross sections are fully correlated, which will be considered in Section 3.2 below.

\subsection{Uncorrelated Total Microscopic Cross Sections}

Considering only the contributions from the group-averaged uncorrelated total microscopic cross sections, the expected value of the leakage response has the expression $[E(L)]_{t}^{(U)}=L\left(\boldsymbol{\alpha}^{0}\right)+[E(L)]_{t}^{(2, U)}$, where the superscript " $(2, \mathrm{U})$ " indicates " $2^{\text {nd }}-$ order, uncorrelated" cross sections, the subscript " $t$ " indicates contributions solely from the group-averaged total microscopic cross sections, and where: (i) $L\left(\boldsymbol{\alpha}^{0}\right)$ denotes the nominal value of the computed leakage response; and (ii) $[E(L)]_{t}^{(2, U)}=\frac{1}{2} \sum_{g=1}^{G} \sum_{i=1}^{I} \frac{\partial^{2} L(\boldsymbol{\alpha})}{\partial \sigma_{t, i}^{g} \partial \sigma_{t, i}^{g}}\left(s_{t, i}^{g}\right)^{2}, G=30, I=6$, where $s_{t, i}^{g}$ denotes the standard deviation associated with the group-averaged total microscopic cross sections $\sigma_{t, i}^{g}$, for each isotope $i$. If, in addition to being uncorrelated, the total microscopic cross sections are also normally-distributed, which will be indicated using the superscript " $(\mathrm{U}, \mathrm{N})$ " then the following expressions hold: (i) the variance, denoted as $[\operatorname{var}(L)]_{t}^{(U, N)}$, of the leakage response of the PERP benchmark becomes $[\operatorname{var}(L)]_{t}^{(U, N)}=[\operatorname{var}(L)]_{t}^{(1, U, N)}+[\operatorname{var}(L)]_{t}^{(2, U, N)}$, where the first-order contribution term is defined as $[\operatorname{var}(L)]_{t}^{(1, U, N)} \triangleq \sum_{g=1}^{G} \sum_{i=1}^{I}\left[\frac{\partial L(\boldsymbol{\alpha})}{\partial \sigma_{t, i}^{g}}\right]^{2}\left(s_{t, i}^{g}\right)^{2}, G=30, I=6$, while the second-order contribution term is defined as: $[\operatorname{var}(L)]_{t}^{(2, U, N)} \triangleq \frac{1}{2} \sum_{g=1}^{G} \sum_{i=1}^{I}\left[\frac{\partial^{2} L(\boldsymbol{\alpha})}{\partial \sigma_{t, i}^{g} \partial \sigma_{t, i}^{g}}\left(s_{t, i}^{g}\right)^{2}\right]^{2}, G=30, I=6 . \quad$ Furthermore, taking into account contributions solely from the group-averaged uncorrelated normally-distributed total microscopic cross sections, the third-order moment, $\left[\mu_{3}(L)\right]_{t}^{(U, N)}$, of the leakage response for the PERP benchmark is $\left[\mu_{3}(L)\right]_{t}^{(U, N)}=3 \sum_{g=1}^{G} \sum_{i=1}^{I}\left[\frac{\partial L(\boldsymbol{\alpha})}{\partial \sigma_{t, i}^{g}}\right]^{2} \frac{\partial^{2} L(\boldsymbol{\alpha})}{\partial \sigma_{t, i}^{g} \partial \sigma_{t, i}^{g}}\left(s_{t, i}^{g}\right)^{4}, G=30, I=6 . \quad$ The skewness, $\left[\gamma_{1}(L)\right]_{t}^{(U, N)}$, of the leakage response, $L$, due to the variances of uncorrelated and normally distributed microscopic total cross sections is defined as $\left[\gamma_{1}(L)\right]_{t}^{(U, N)}=\left[\mu_{3}(L)\right]_{t}^{(U, N)} /\left\{[\operatorname{var}(L)]_{t}^{(U, N)}\right\}^{3 / 2}$. If the $2^{\text {nd }}$ order sensitivities were unavailable, the third moment $\left[\mu_{3}(L)\right]_{t}^{(U, N)}$ and hence the skewness $\gamma_{1}$ of the leakage response would vanish and the response distribution would by default be assumed to be Gaussian.

\subsection{Fully Correlated Total Microscopic Cross Sections}

The effects of correlations among the group-averaged microscopic total cross sections, and hence the impact of the second-order mixed sensitivities of the leakage response to these cross sections, can also be assessed in the extreme case of fully correlated cross sections. Thus, if the group-averaged microscopic total cross sections were fully correlated (denoted by using the superscript " $F C$ ", the expectation value of 
the leakage response would be given by the expression $[E(L)]_{t}^{(F C)}=L\left(\boldsymbol{\alpha}^{0}\right)+[E(L)]_{t}^{(2, F C)}$, where the quantity $[E(L)]_{t}^{(2, F C)}$ denotes the contributions from both the unmixed and mixed $2^{\text {nd }}$-order sensitivities when the total cross sections parameters are fully correlated, and is given by the following expression: $[E(L)]_{t}^{(2, F C)} \triangleq \frac{1}{2} \sum_{g=1}^{G} \sum_{g^{\prime}=1}^{G} \sum_{i=1}^{I} \sum_{k=1}^{I} \frac{\partial^{2} L(\boldsymbol{\alpha})}{\partial \sigma_{t, i}^{g} \partial \sigma_{t, k}^{g^{\prime}}}\left(s_{t, i}^{g} s_{t, k}^{g^{\prime}}\right), \quad G=30, I=6$. The additional $2^{\text {nd }}$-order contributions to the expectation value of the leakage response when the total cross sections are fully correlated is denoted as $[E(L)]_{t}^{(2, M S C)}$, where the superscript "MSC" denotes "mixed second-order correlated," and is computed using the following expression: $[E(L)]_{t}^{(2, M S C)}=[E(L)]_{t}^{(2, F C)}-[E(L)]_{t}^{(2, U)}$. If the total microscopic cross sections were fully correlated and normally-distributed [indicated by the superscript " $(F C, N)$ "], the variance of the leakage response would be given by the expression $[\operatorname{var}(L)]_{t}^{(F C, N)}=[\operatorname{var}(L)]_{t}^{(1, F C, N)}+[\operatorname{var}(L)]_{t}^{(2, F C, N)} \quad, \quad$ where: (i) the quantity $[\operatorname{var}(L)]_{t}^{(1, F C, N)} \triangleq \sum_{g=1}^{G} \sum_{g^{\prime}=1}^{G} \sum_{i=1}^{I} \sum_{k=1}^{I}\left[\frac{\partial L(\boldsymbol{\alpha})}{\partial \sigma_{t, i}^{g}} \frac{\partial L(\boldsymbol{\alpha})}{\partial \sigma_{t, k}^{g^{\prime}}}\right]\left(s_{t, i}^{g} s_{t, k}^{g^{\prime}}\right)$ denotes the contributions from the $1^{\text {st }}$-order sensitivities when the total cross sections parameters are fully correlated; and (ii) the quantity $[\operatorname{var}(L)]_{t}^{(2, F C, N)} \triangleq \frac{1}{2}\left[\sum_{g=1}^{G} \sum_{g^{\prime}=1}^{G} \sum_{i=1}^{I} \sum_{k=1}^{I} \frac{\partial^{2} L(\boldsymbol{\alpha})}{\partial \sigma_{t, i}^{g} \partial \sigma_{t, k}^{g^{\prime}}} s_{t, i}^{g} s_{t, k}^{g^{\prime}}\right]\left[\sum_{g=1}^{G} \sum_{g^{\prime}=1}^{G} \sum_{\mu=1}^{I} \sum_{v=1}^{I} \frac{\partial^{2} L(\boldsymbol{\alpha})}{\partial \sigma_{t, \mu}^{g} \partial \sigma_{t, v}^{g^{\prime}}} s_{t, \mu}^{g} s_{t, v}^{g^{\prime}}\right]$ denotes the contributions from both the mixed and unmixed $2^{\text {nd }}$-order sensitivities, with $G=30, I=6$. The contributions to $[\operatorname{var}(L)]_{t}^{(F C, N)}$ involving the first-order sensitivities will be denoted as $[\operatorname{var}(L)]_{t}^{(1, M S C)}$, where the superscript "(1, MSC)" denotes "first-order, mixed sensitivities, correlated," and are obtained by subtracting the uncorrelated terms from the fully correlated ones, i.e., $[\operatorname{var}(L)]_{t}^{(1, M S C)}=[\operatorname{var}(L)]_{t}^{(1, F C, N)}-[\operatorname{var}(L)]_{t}^{(1, U, N)}$. The contributions to $[\operatorname{var}(L)]_{t}^{(F C, N)}$ involving the second-order sensitivities will be denoted as $[\operatorname{var}(L)]_{t}^{(2, M S C)}$ and are obtained by subtracting the respective uncorrelated terms from the respective fully correlated ones, i.e., $[\operatorname{var}(L)]_{t}^{(2, M S C)}=[\operatorname{var}(L)]_{t}^{(2, F C)}-[\operatorname{var}(L)]_{t}^{(2, U, N)}$.

For fully correlated total cross sections, the third-order cumulant has the following expression:

$$
\begin{aligned}
& {\left[\mu_{3}(L)\right]_{t}^{(F C, N)}=\left[\sum_{g=1}^{G} \sum_{g^{\prime}=1}^{G} \sum_{i=1}^{I} \sum_{k=1}^{I} \frac{\partial L(\boldsymbol{\alpha})}{\partial \sigma_{t, i}^{g}} s_{t, i}^{g} \frac{\partial L(\boldsymbol{\alpha})}{\partial \sigma_{t, k}^{g^{\prime}}} s_{t, k}^{g^{\prime}}\right]\left[\sum_{g=1}^{G} \sum_{g^{\prime}=1}^{G} \sum_{\mu=1}^{I} \sum_{v=1}^{I} \frac{\partial^{2} L(\boldsymbol{\alpha})}{\partial \sigma_{t, \mu}^{g} \partial \sigma_{t, v}^{g^{\prime}}} s_{t, \mu}^{g} s_{t, v}^{g^{\prime}}\right]} \\
& +\left[\sum_{g=1}^{G} \sum_{g^{\prime}=1}^{G} \sum_{i=1}^{I} \sum_{v=1}^{I} \frac{\partial L(\boldsymbol{\alpha})}{\partial \sigma_{t, i}^{g}} s_{t, i}^{g} \frac{\partial L(\boldsymbol{\alpha})}{\partial \sigma_{t, v}^{g^{\prime}}} s_{t, v}^{g^{\prime}}\right]\left[\sum_{g=1}^{G} \sum_{g^{\prime}=1}^{G} \sum_{k=1}^{I} \sum_{\mu=1}^{I} \frac{\partial^{2} L(\boldsymbol{\alpha})}{\partial \sigma_{t, k}^{g^{\prime}} \partial \sigma_{t, \mu}^{g}} s_{t, k}^{g^{\prime}} s_{t, \mu}^{g}\right] \\
& +\left[\sum_{g=1}^{G} \sum_{g^{\prime}=1}^{G} \sum_{i=1}^{I} \sum_{k=1}^{I} \frac{\partial^{2} L(\boldsymbol{\alpha})}{\partial \sigma_{t, i}^{g} \partial \sigma_{t, k}^{g^{\prime}}} s_{t, i}^{g} s_{t, k}^{g^{\prime}}\right]\left[\sum_{g=1}^{G} \sum_{g^{\prime}=1}^{G} \sum_{\mu=1}^{I} \sum_{v=1}^{I} \frac{\partial L(\boldsymbol{\alpha})}{\partial \sigma_{t, \mu}^{g}} s_{t, \mu}^{g} \frac{\partial L(\boldsymbol{\alpha})}{\partial \sigma_{t, v}^{g^{\prime}}} s_{t, v}^{g^{\prime}}\right], \text { for } G=30, I=6 .
\end{aligned}
$$

The contributions stemming from the mixed $2^{\text {nd }}$-order sensitivities when the total cross sections are fully correlated and normally distributed will be denoted as $\left[\mu_{3}(L)\right]_{t}^{(M S C, N)}$, where the superscript " $(M S C, N)$ "

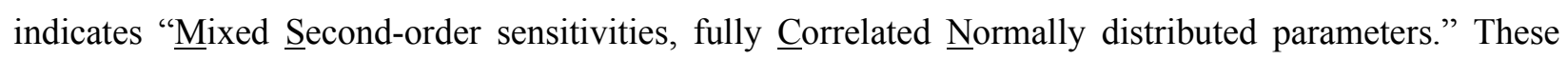


contributions are computed by subtracting the uncorrelated terms, $\left[\mu_{3}(L)\right]_{t}^{(U, N)}$, from the respective fully correlated ones, $\left[\mu_{3}(L)\right]_{t}^{(F C, N)}$, to obtain: $\left[\mu_{3}(L)\right]_{t}^{(M S C, N)}=\left[\mu_{3}(L)\right]_{t}^{(F C, N)}-\left[\mu_{3}(L)\right]_{t}^{(U, N)}$. For fully correlated total cross sections, the skewness, $\left[\gamma_{1}(L)\right]_{t}^{(F C, N)}$, is defined as usual: $\left[\gamma_{1}(L)\right]_{t}^{(F C, N)}=\left[\mu_{3}(L)\right]_{t}^{(F C, N)} /\left\{[\operatorname{var}(L)]_{t}^{(F C, N)}\right\}^{3 / 2}$.

The effects of the first- and second-order sensitivities on the response moments (expected value, variance and skewness) can be highlighted by considering uniform values for the standard deviations of the group-averaged isotopic total microscopic cross sections and using these values together with the respective sensitivities. The results thus obtained are presented in Table 4, for illustrative uniform relative standard deviations for the parameters of $5 \%$ and $10 \%$, respectively, for the standard deviations $s_{t, i}^{g}$.

Table 4 Response moments for various relative standard deviations of total microscopic cross section which are fully correlated.

\begin{tabular}{|ccc|lll|}
\hline \multicolumn{2}{|c|}{ Fully Correlated Cross Sections } & \multicolumn{3}{c|}{ Uncorrelated Cross Sections } \\
\hline Rel. St. Dev. & $\mathbf{1 0 \%}$ & $\mathbf{5 \%}$ & Rel. St. Dev. & $\mathbf{1 0 \%}$ & $\mathbf{5 \%}$ \\
\hline$L\left(\boldsymbol{\alpha}^{0}\right)$ & $1.7648 \times 10^{6}$ & $1.7648 \times 10^{6}$ & $L\left(\boldsymbol{\alpha}^{0}\right)$ & $1.7648 \times 10^{6}$ & $1.7648 \times 10^{6}$ \\
\hline$[E(L)]_{t}^{(2, F C)}$ & $2.9451 \times 10^{7}$ & $7.3627 \times 10^{6}$ & {$[E(L)]_{t}^{(2, U)}$} & $4.5980 \times 10^{6}$ & $1.1495 \times 10^{6}$ \\
\hline$[E(L)]_{t}^{(2, M S C)}$ & $2.4853 \times 10^{7}$ & $6.2132 \times 10^{6}$ & {$[E(L)]_{t}^{(U)}$} & $6.3628 \times 10^{6}$ & $2.9143 \times 10^{6}$ \\
\hline$[E(L)]_{t}^{(F C)}$ & $3.1216 \times 10^{7}$ & $9.1275 \times 10^{6}$ & {$[\operatorname{var}(L)]_{t}^{(1, U, N)}$} & $3.4196 \times 10^{12}$ & $8.5490 \times 10^{11}$ \\
\hline$[\operatorname{var}(L)]_{t}^{(1, F C, N)}$ & $4.7601 \times 10^{13}$ & $1.1900 \times 10^{13}$ & {$[\operatorname{var}(L)]_{t}^{(2, U, N)}$} & $2.8789 \times 10^{13}$ & $1.7993 \times 10^{12}$ \\
\hline$[\operatorname{var}(L)]_{t}^{(1, M S C)}$ & $4.4181 \times 10^{13}$ & $1.1045 \times 10^{13}$ & {$[\operatorname{var}(L)]_{t}^{(U, N)}$} & $3.2208 \times 10^{13}$ & $2.6542 \times 10^{12}$ \\
\hline$[\operatorname{var}(L)]_{t}^{(2, F C, N)}$ & $1.7347 \times 10^{15}$ & $1.0842 \times 10^{14}$ & {$\left[\mu_{3}(L)\right]_{t}^{(U, N)}$} & $6.2267 \times 10^{19}$ & $3.8917 \times 10^{18}$ \\
\hline$[\operatorname{var}(L)]_{t}^{(2, M S C)}$ & $1.7059 \times 10^{15}$ & $1.0662 \times 10^{14}$ & {$\left[\gamma_{1}(L)\right]_{t}^{(U, N)}$} & 0.3407 & 0.8999 \\
\hline$[\operatorname{var}(L)]_{t}^{(F C, N)}$ & $1.7823 \times 10^{15}$ & $1.2302 \times 10^{14}$ & & & \\
\hline$\left[\mu_{3}(L)\right]_{t}^{(F C, N)}$ & $8.4113 \times 10^{21}$ & $5.2571 \times 10^{20}$ & & & \\
\hline$\left[\mu_{3}(L)\right]_{t}^{(M S C, N)}$ & $8.3490 \times 10^{21}$ & $5.2181 \times 10^{20}$ & & & \\
\hline$\left[\gamma_{1}(L)\right]_{t}^{(F C, N)}$ & 0.1118 & 0.3983 & & & \\
\hline
\end{tabular}

As indicated in Table 4, the trends displayed by the results for a uniform standard deviation of 5\% for the group isotopic microscopic cross sections are amplified significantly when this uniform standard deviation is increased to $10 \%$. Thus, the $2^{\text {nd }}$-order contribution $[E(L)]_{t}^{(2, U)}$ for uncorrelated cross sections is $260 \%$ larger than the computed leakage value $L\left(\boldsymbol{\alpha}^{0}\right)$, contributing $72 \%$ of the expected value for the leakage response $[E(L)]_{t}^{(U)}$ by comparison to $28 \%$ contributed by the computed value $L\left(\boldsymbol{\alpha}^{0}\right)$. For fully correlated cross sections, the results presented in Table 4 indicate that $[E(L)]^{(2, F C)}>6 \times[E(L)]_{t}^{(2, U)}$, highlighting the very significant impact of the mixed $2^{\text {nd }}$-order sensitivities on causing the expected value 
of the leakage response to differ significantly from the computed response value, since, as shown by the results presented in Table $4,[E(L)]_{t}^{(F C)} \cong 20 \times L\left(\boldsymbol{\alpha}^{0}\right) \cong 5 \times[E(L)]_{t}^{(U)}$. Thus, the customary procedure of neglecting second (and higher) order sensitivities and considering that the computed value, $L\left(\boldsymbol{\alpha}^{0}\right)$, as the actual expected (i.e., mean) value of the distribution, would be in error, from $360 \%$ to $2000 \%$.

Furthermore, for uncorrelated cross sections, the contribution $[\operatorname{var}(L)]_{t}^{(2, U, N)}$ of the unmixed $2^{\text {nd }}-$ order sensitivities to the response variance $[\operatorname{var}(L)]_{t}^{(U)}$ is $847 \%$ larger than the contribution $[\operatorname{var}(L)]_{t}^{(1, U, N)}$ stemming from the first-order sensitivities. Hence, neglecting these $2^{\text {nd }}-$ order contributions would cause a very large non-conservative error by under-reporting of the response variance by a factor of $947 \%$. The results shown in Table 4 also indicate that $[\operatorname{var}(L)]_{t}^{(2, F C, N)} \cong 60 \times[\operatorname{var}(L)]_{t}^{(2, U, N)}$, with the consequence that $[\operatorname{var}(L)]_{t}^{(F C, N)} \cong 60 \times[\operatorname{var}(L)]_{t}^{(U)}$, thus demonstrating that the mixed $2^{\text {nd }}$-order sensitivities are extremely important in contributing to the leakage response variance, when the cross sections are imprecisely known. The results presented in Table 4 also indicate the effect of the correlations induced by the mixed $2^{\text {nd }}$-order sensitivities is to render the leakage response distribution to be more symmetrical about the response's expected value.

\section{CONCLUSIONS}

Even when the group-averaged microscopic total cross sections are uncorrelated, the results in Table 4 indicate that the importance of the $2^{\text {nd }}$-order sensitivities relative to the importance of the $1^{\text {st }}$-order ones increases as the parameters uncertainties increase. The effects of the $2^{\text {nd }}$-order sensitivities are to increase the expected value of the response versus the computed response value, which shifts to positive values the distribution of the leakage response in parameters space. Also, the contributions of the $2^{\text {nd }}$-order sensitivities to the response's variance overtake the contributions of the $1^{\text {st }}$-order sensitivities to the response's variance already for relatively small (ca. 5\%) parameter standard deviations. These effects are rapidly amplified when the parameters are less precisely known. In particular, it has been shown that, for a uniform standard deviation of $10 \%$, the $2^{\text {nd }}$-order sensitivities contribute $72 \%$ of the expected value for the leakage response $E(L)$ by comparison to $28 \%$ contributed by the computed value $L\left(\boldsymbol{\alpha}^{0}\right)$. Furthermore, the contribution $[\operatorname{var}(L)]^{(2, U, N)}$ of the second-order sensitivities to the response variance $\operatorname{var}(L)$ is $847 \%$ larger than the contribution $[\operatorname{var}(L)]^{(1, U, N)}$ stemming from the first-order sensitivities. Thus, the customary procedure of neglecting second (and higher) order sensitivities and considering that the computed value, $L\left(\boldsymbol{\alpha}^{0}\right)$, is the actual expected (i.e., mean) value, $E(L)$, of the distribution, would be in error by $362 \%$. Hence, neglecting these second-order contributions would cause a very large nonconservative error by under-reporting of the response variance by a factor of $947 \%$. In all cases, the second-order sensitivities cause the leakage distribution in parameter space to be skewed to positive values relative to the expected value, which, in turn, is significantly shifted to much larger positive values than the computed leakage $L\left(\boldsymbol{\alpha}^{0}\right)$. All in all, neglecting the second-order sensitivities would erroneously predict a Gaussian distribution for the leakage distribution in parameter space, centered about the computed leakage $L\left(\boldsymbol{\alpha}^{0}\right)$.

The results presented in Table 4 also indicate that the mixed $2^{\text {nd }}$-order sensitivities play a very significant role in determining the moments of the leakage response distribution for correlated cross sections. The importance of the mixed $2^{\text {nd }}$-order sensitivities increases as the relative standard deviations for the cross sections increase. For fully correlated cross sections, for example, neglecting the $2^{\text {nd }}$-order sensitivities 
would cause an error as large as $2000 \%$ in the expected value of the leakage response, and up to $6000 \%$ in the variance of the leakage response.

Of course, neither the fully uncorrelated nor the fully correlated illustrative examples presented in this work realistically describe the actual physical situations regarding the total microscopic cross section parameters. The fully uncorrelated case underestimates reality while the fully correlated case overestimates it. In reality, total cross sections are partially correlated, so reality falls in between the fully uncorrelated and fully correlated cases. However, correlations among cross sections are scantly available at this time in the evaluated nuclear data files. The purpose of this work is to draw attention to the fact that $2^{\text {nd }}$-order sensitivities are important, and their effects must be assessed for each physical system under consideration. In particular for the PERP benchmark, it has been shown that the $2^{\text {nd }}$-order sensitivities are even more important than the $1^{\text {st }}$-order ones. While the effects of the $2^{\text {nd }}$-order sensitivities may be less marked for other reactor physics systems, the point is that they are not always negligible, as they have been considered in hitherto in the published literature. Furthermore, the effects of the mixed $2^{\text {nd }}$-order sensitivities, as illustrated for the PERP benchmark in this work, underscore the need for future experimental research aimed at obtaining values for the correlations that might exist among the total cross sections, which are unavailable at this time.

The complete $2^{\text {nd }}$-order sensitivity analysis of the PERP benchmark can be found in References 7 - 12 .

\section{REFERENCES}

1. International Handbook of Evaluated Criticality Safety Benchmark Experiments, Nuclear Energy Agency. - Paris: OECD Nuclear Energy Agency. 2016. (NEA; 7328).

2. E. Brandon, "Assembly of 239Pu Ball for Criticality Experiment," CMB-11-FAB-80-65, Los Alamos National Laboratory (1980).

3. E. C. Miller, et al, "Computational Evaluation of Neutron Multiplicity Measurements of Polyethylene-Reflected Plutonium Metal," Nucl. Sci. Eng., 176(2), 167-185 (2014).

4. D. G. Cacuci, "Application of the Second-Order Comprehensive Adjoint Sensitivity Analysis Methodology to Compute 1st- and 2nd-Order Sensitivities of Flux Functionals in a Multiplying System with Source," Nucl. Sci. Eng, 193, 555-600 (2019).

5. R. E. Alcouffe, R. S. Baker, J. A. Dahl, S.A. Turner, and R. Ward, "PARTISN: A Time-Dependent, Parallel Neutral Particle Transport Code System," LA-UR-08-07258 (Revised Nov. 2008).

6. J. L. Conlin, et al., "MENDF71X: Multigroup Neutron Cross-Section Data Tables Based upon ENDF/B-VII.1X," Los Alamos National Laboratory report LA-UR-15-29571 (October 7, 2013);

7. Cacuci, D.G.; Fang, R.; Favorite, J.A., Comprehensive Second-Order Adjoint Sensitivity Analysis Methodology (2 $\left.2^{\text {nd }}-A S A M\right)$ Applied to a Subcritical Experimental Reactor Physics Benchmark: I. Effects of Imprecisely Known Microscopic Total and Capture Cross Sections, Energies, 12, 4219, 2019.

8. Fang, R.; Cacuci, D.G., Comprehensive Second-Order Adjoint Sensitivity Analysis Methodology ( $\left.2^{\text {nd }}-A S A M\right)$ Applied to a Subcritical Experimental Reactor Physics Benchmark: II. Effects of Imprecisely Known Microscopic Scattering Cross Sections. Energies, 12, 4114, 2019.

9. Cacuci, D.G.; Fang, R.; Favorite, J.A.; Badea, M.C.; di Rocco, F., Comprehensive Second-Order Adjoint Sensitivity Analysis Methodology (2nd $-A S A M)$ Applied to a Subcritical Experimental Reactor Physics Benchmark: III. Effects of Imprecisely Known Microscopic Fission Cross Sections and Average Number of Neutrons per Fission. Energies, 12, 4100, 2019.

10. Fang, R.; Cacuci, D.G., Comprehensive Second-Order Adjoint Sensitivity Analysis Methodology ( $\left.2^{\text {nd }}-A S A M\right)$ Applied to a Subcritical Experimental Reactor Physics Benchmark: IV. Effects of Imprecisely Known Source Parameters. Energies, 2020, submitted. 
11. Fang, R.; Cacuci, D.G., Comprehensive Second-Order Adjoint Sensitivity Analysis Methodology ( $\left.2^{\text {nd }}-A S A M\right)$ Applied to a Subcritical Experimental Reactor Physics Benchmark: V. Computation of Mixed 2 ${ }^{\text {nd }}$-Order Sensitivities Involving Isotopic Number Densities. Energies, 2020, submitted.

12. Cacuci, D.G.; Fang, R.; Favorite, J.A., Comprehensive Second-Order Adjoint Sensitivity Analysis Methodology (2 $\left.2^{\text {nd }}-A S A M\right)$ Applied to a Subcritical Experimental Reactor Physics Benchmark. VI: Overall Impact of $1^{\text {st }}$ - and $2^{\text {nd }}$-Order Sensitivities on Response Uncertainties. Energies, 2020, submitted. 